\title{
Icing Prediction of Fan Blade based on a Hybrid Model
}

\author{
Cheng Peng ${ }^{\mathrm{a}, \mathrm{b}}$, Jing $\mathrm{He}^{\mathrm{a}}$, Hao Chi ${ }^{\mathrm{a}}$, Xinpan Yuan ${ }^{\mathrm{a}, \mathrm{b}}$, and Xiaojun Deng ${ }^{\mathrm{a}, *}$ \\ ${ }^{a}$ School of Computer Science, Hunan University of Technology, Zhuzhou, 412007, China \\ ${ }^{b}$ School of Automation, Central South University, Changsha, 410083, China
}

\begin{abstract}
For the problem that fan blade icing failures cannot be accurately predicted in advance, a data-driven fault prediction method is proposed in this paper. Firstly, the delay window is introduced to the PCA algorithm to extract the fault mode related features from the SCADA high-dimensional data. Then, the trained Elman neural network is adopted to predict the future value of the relevant features. Finally, a BP self-clustering algorithm is designed to predict the icing fault of the blade with the multi-source data fusion. The results show that the proposed method can effectively predict the icing failure of wind turbine blades and has reference significance for the maintenance of wind turbines.
\end{abstract}

Keywords: wind turbine; fault prediction; blade icing; neural network; feature extraction; data-driven

(Submitted on September 19, 2019; Revised on September 30, 2019; Accepted on October 25, 2019)

(C) 2019 Totem Publisher, Inc. All rights reserved.

\section{Introduction}

According to statistics, in 2016, the world's new installed wind power capacity exceeded $54.6 \mathrm{GW}$, and the cumulative installed capacity reached $486.8 \mathrm{GW}$. China ranks first in the world in both of cumulative installed capacity and new installed capacity of wind power [1]. However, with the rapid development of wind power, the frequent failure of wind turbines has brought about high operating and maintenance costs. Therefore, research on wind turbine fault prediction is of great significance to improve the reliability and economy of wind turbines.

After years of research on wind turbine fault diagnosis and health management technology, prognostic and health management (PHM) has been a hot spot both at home and abroad [2]. However, research on the icing failure of wind turbine blades is relatively rare. Blade icing is a very complex nonlinear problem that is difficult to model accurately. In view of the above problems, this paper intends to use data drive technology to mine SCADA (supervisory control and data acquisition) data and establish a blade icing failure prediction model to achieve accurate prediction of blade icing failure. This transforms the past stress-type maintenance method into an active predictive maintenance method, which in turn improves the operation and maintenance costs of wind turbines [3].

There are many wind turbines installed in high-altitude windy areas, with blade icing failures. These environments can affect power generation efficiency, accelerate fan wear, break fan blades, and pose dangers to power plant personnel and power equipment. In recent years, these issues have been addressed through additional research arrangements of ultrasound, infrared, and other sensors. However, these measures are only for emergency treatment after failure, and due to the need for extra customization and operating costs, the penetration rate in practical applications is also very low. At the same time, although many new wind turbines have been designed with automatic deicing systems, the practical problem is to accurately predict the early process of icing and open the de-icing system at the right time. Therefore, improving the accuracy of the automatic de-icing system for blade icing prediction is an urgent problem to be solved [4]. Blade fault prediction and diagnosis have been studied at home and abroad. For example, Alzghoul et al. tested and compared different linear regression prediction methods based on data flow in the developed fault detection system. The results showed that the linear regression method has good performance in short-term data prediction. The best performance involved a mean absolute

\footnotetext{
* Corresponding author.

E-mail address: chengpeng@ csu.edu.cn
} 
error (MAE) of around 0.4 and a prediction accuracy of 87.5\% [5]. Kusiak et al. proposed the use of data mining to predict the failure of wind turbines [6]. Zhang proposed to build a system to deal with icing faults based on the fluctuation of longterm real-time data of the collecting fan [7]. Shi summarized the methods of research on existing blade icing failures [8]. However, although the above methods have obvious advantages in terms of physical significance, they generally only select a kind of monitoring data with a high intuitive correlation with blade icing faults for prediction, instead of using the potential complementarities of existing multi-source data in wind farms to conduct fusion mining to obtain more practical and more accurate values. The innovation of this research is based on the data set collected by the SCADA system, which has been popularized by a large number of wind farms. The fault prediction of wind turbine blade icing based on multisource data fusion seeks to not only reduce the maintenance costs of wind turbine icing failure, but also provide a new idea for the prediction of wind turbine blade failure.

\section{Research on Prediction Methods}

This section gives the structure and steps of the prediction process proposed in this paper and introduces the three key theoretical methods used.

\subsection{Predicting Process}

In the view of dynamic characteristics of the wind turbine blade icing failure, this paper innovatively proposes the prediction process as shown in the following figure. Firstly, the SCADA monitoring data set is extracted for relevance feature extraction. Then, the Elman neural network is used to establish the prediction sub-model for the extracted information, and the sub-model prediction results are multi-source data fusion. Finally, the BP neural network trained according to a large number of historical data sets is used to predict the icing of the self-clustering leaves. The improved dynamic PCA algorithm will better screen out the multi-class monitoring data with the highest correlation with icing faults, which reduces the difficulty of analyzing problems. At the same time, it fully considers the advantage of the Elman neural network in dynamic modeling, which is beneficial to the accuracy of prediction of wind blade icing failure. The specific process is shown in Figure 1.

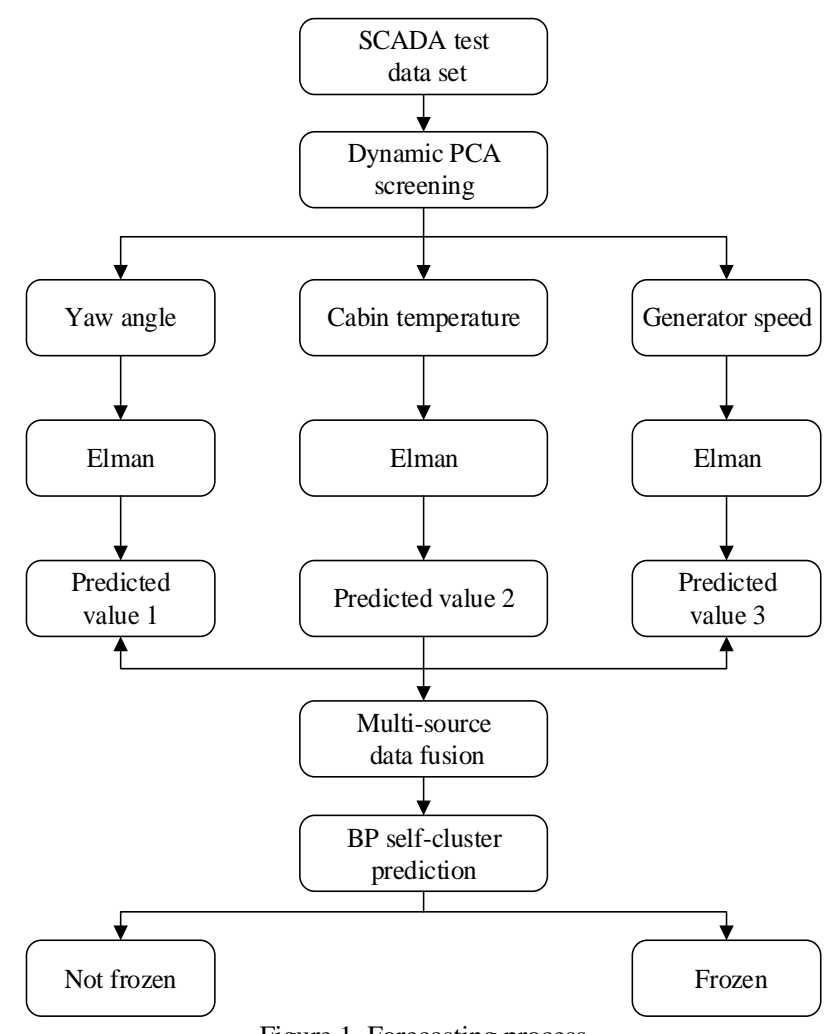

Figure 1. Forecasting process

\subsection{Improved PCA Algorithm}

In order to reduce the feature dimension of SCADA monitoring data, this paper uses the PCA (principal component analysis) 
algorithm in the multivariate statistical analysis algorithm. It uses the idea of coordinate transformation to transform highdimensional space problems into low-dimensional space processing, which makes the problem simpler and more intuitive [9]. However, the data $X$ trained in the PCA feature extraction method has only two-dimensional data of variables and sampling times, which assumes that the observation data at a certain moment is independent of the historical observation data sequence. This assumption is valid when the sampling interval is long, but when the sampling interval is short, in addition to considering the cross-correlation between different variables, the autocorrelation needs to be considered. The improved method uses dynamic PCA technology.

The dynamic PCA feature extraction method constructs the dynamic time data by using the dynamic time series of the static data of the original variable and then uses the PCA method to extract the features. The dynamic relationship between the system variables can thereby be effectively extracted, so as to accurately describe the dynamic behavior of the system. Suppose that the observation data set $X$ under normal conditions is sampled, it contains $m$ observation variables, each variable has $n$ observations, and $X$ can be expressed as Equation (1).

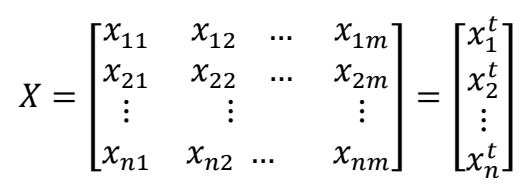

Where $x_{i} \in R^{m}(i=1,2, \cdots, n)$ is an $m$-dimensional vector. $X$ is extended according to the idea of dynamic time series, that is, the $h$ observations in front of a certain time $t$ are expanded for each observation vector, as shown in Equation (2).

$$
X(h)=\left[\begin{array}{cccc}
x_{t}^{T} & x_{t-1}^{T} & \cdots & x_{t-h}^{T} \\
x_{t-1}^{T} & x_{t-2}^{T} & \cdots & x_{t-h-1}^{T} \\
\vdots & \vdots & & \vdots \\
x_{t-n+h}^{T} & x_{t-n+h-1}^{T} & \cdots & x_{t-n}^{T}
\end{array}\right]
$$

Where $x_{t}^{\mathrm{T}}$ is the $m$-dimensional observation vector in the training set at time $t$. The method of calculating the delay window $h$ is as follows: first, the static condition of $h=0$ is processed, and the number of static relations in the data matrix is equal to the difference between the number of variables and the number of principal elements. Then, let $h=1$, and calculate a new dynamic relationship number. The new dynamic relationship number is equal to the number of variables minus the number of principal elements and the number of static relations calculated in step 1 , and the $h$ function is gradually increased. The new relational function $r_{\text {new }}(h)$ is recursively calculated and expressed as Equation (3).

$$
r_{\text {new }}(h)=r(h)-\sum_{i=0}^{h-1}(h-i+1) r_{\text {new }}(i)
$$

The process is continued until there is no new static and dynamic relationship and $r_{\text {new }}(h) \leq 0$.

The process of extracting features using dynamic PCA is actually a process of extracting PCA features for augmented matrices. The specific steps are as follows:

- Step 1. Collect data.

- Step 2. Calculate the delay window according to Equation (3).

- Step 3. Construct a dynamic data matrix according to Equation (2), and convert the matrix into a standard data matrix with a mean of 0 and a variance of 1 .

- Step 4. $\Sigma=\frac{1}{n-1} X^{\mathrm{T}} X$. Calculate the covariance matrix $\Sigma$.

- Step 5. Calculate the principal component variance vector $\left[\lambda_{1}, \lambda_{2}, \cdots, \lambda_{m}\right]^{\mathrm{T}}$ and the corresponding load matrix $\left[p_{1}, p_{2}, \cdots, p_{m}\right]$.

- Step 6. According to the cumulative and percentage of variance (CPV), use $C P V=\sum_{i=1}^{k} \lambda_{i} / \sum_{i=1}^{m} \lambda_{i}$ to determine the number of principals; generally, take $C P V \geq 85 \%$

- Step 7. According to the specific experimental needs, if the number of principal elements $k$ is selected, the first $k$ features are identified as the main component. 


\subsection{Elman Neural Network}

The Elman neural network is a typical dynamic recurrent neural network with a simple structure and superior performance. Therefore, it is used to predict various monitoring values. The structure is shown in the following figure [10]. In addition to the input layer, the hidden layer, and the output layer, the Elman network also has a special context layer, which is used to memorize the hidden layer unit. The output value is a delay operator that enables the network to have dynamic memory functions [11]. The trained Elman network has nonlinear mapping and dynamic characteristics, which is suitable for reflecting the dynamic characteristics of wind turbine blade icing failure. The mathematical model of the Elman neural network is shown in Equation (4).

$$
\begin{gathered}
x(k)=f\left(w^{I 1} x_{c}(k)+w^{I 2} u(k-1)\right) \\
x_{c}(k)=x(k-1) \\
y(k)=\mathrm{g}\left(w^{I 3} x(k)\right)
\end{gathered}
$$

Among them, the connection weight $w^{I 1}$ is a connection weight matrix of the contact unit and the hidden layer unit, $w^{I 2}$ is a connection weight matrix of the input unit and the hidden layer unit, $w^{I 3}$ is a connection weight matrix of the hidden layer unit and the output unit, $x_{c}(k)$ and $x(k)$ respectively represent the output of the contact unit and the hidden layer unit, $y(k)$ represents the output of the output unit, and $f(\cdot)$ and $g(\cdot)$ are the transfer functions of the hidden layer unit and the output unit, respectively.

\subsection{BP Neural Networks}

Self-cluster prediction based on multi-source eigenvalues predicted by the Elman neural network can be understood as a nonlinear system, and the BP neural network is a kind of nonlinear optimization. It adjusts the weights and thresholds of the network according to the error back propagation so that the sum of the system errors is the smallest [12-13]. Kolmogorov's theorem states that the three layers of the BP neural network are sufficient to approximate complex nonlinear systems with arbitrary precision [14-16]. Therefore, this paper adopts a three-layer BP neural network. The number of input nodes is $M=$ 3 , the number of output nodes is $N=1$, and the number of nodes in the hidden layer of training is seven. The structure of the BP neural network is shown in Figure 2.

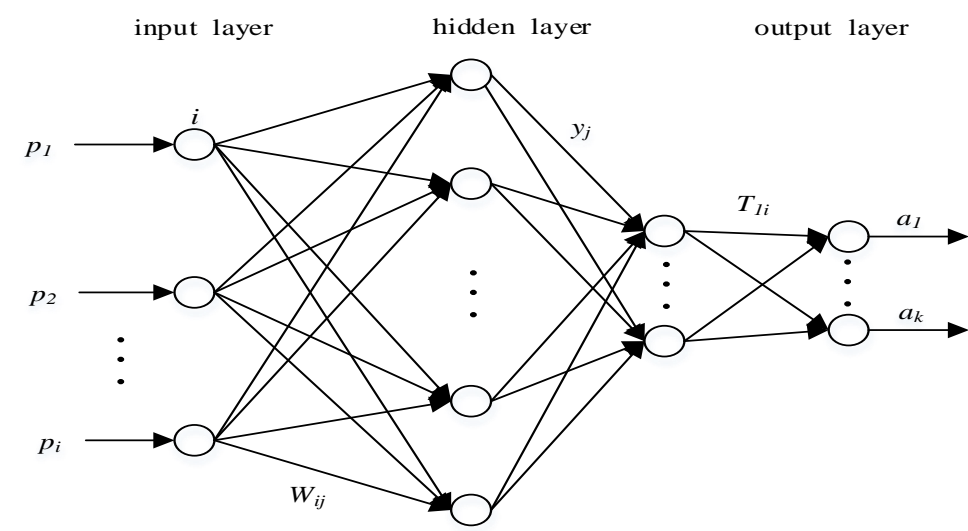

Figure 2. BP neural network structure

In the above figure, $x_{i}^{n}$ is the $i^{\text {th }}$ input of the $n^{\text {th }}(n=1,2,3, \cdots)$ input layers $(i=1,2,3, \cdots, m), y_{i}^{n}$ is the $n^{\text {th }}$ output of the layer $l$, and this article takes $n=3 . H$ is the number of hidden layer nodes trained, $W_{i h}$ is the weight of the input layer to the hidden layer connection, and $W_{h k}$ is the weight of the output layer to the hidden layer connection. The purpose of the algorithm is to determine the parameters $W_{i h}$ and $W_{h k}$ after fitting optimally so that the two sequences $y_{i}^{n}$ and $d_{l}^{n}$ are optimally fitted. $d_{l}^{n}$ is the $l^{\text {th }}$ expected output value of the $n^{\text {th }}$ sample. The error means the square value is $E=\sum\left(d_{i}^{n}-y_{i}^{n}\right)^{2} y_{i}^{n}$.

\section{Case Study}

\subsection{SCADA Data Preprocessing}

The data used in this paper is derived from SCADA data (sampling interval of $7 \mathrm{~s}$ ) and state data collected by a $2.5 \mathrm{MW}$ wind 
turbine in Inner Mongolia from a standardized SCADA system in November 2015, during which multiple blade icing failures occurred. The SCADA data mainly provides the historical operation information of the wind turbine, and the status data mainly provides the fault status information of the wind turbine.

From the SCADA data, the wind speed, power, pitch angle, temperature, and 26 other characteristic parameters that characterize the operating conditions and operating environment of the wind turbine are extracted, and the data within $1 \mathrm{~min}$ is averaged. A total of 37398 groups of records are obtained, as shown in the following two tables. According to the state data of the wind turbines, the normal state and the fault state are represented by 0 and 1 , respectively.

\subsection{Feature Filtrateing}

The SCADA system records a large number of characteristic parameters to monitor the fan performance, but not all parameters of SCADA data are related to the failure modes studied. In addition, redundancy between feature parameters in SCADA data may affect the classification performance. Therefore, it is necessary to evaluate the classification ability of each feature parameter, select the feature parameters with strong classification ability, and eliminate redundant information to reduce the feature dimension.

In this paper, the data is analyzed by principal component analysis (PCA) with a delay window to detect and select the characteristics that are highly correlated with the fan blades [17]. The following Table 1 shows the results after screening. As the load values of various types of monitoring data decrease rapidly, only a few characteristic parameters with large load values are selected, as shown in the following table. At the same time, it is concluded that the characteristic parameters with high correlation with blade icing fault include the yaw angle, cabin temperature, ambient temperature, generator speed, and power [18]. Since the redundancy in the characteristics will affect the performance of the model, this study selects monitoring data such as the yaw angle, cabin temperature, and generator speed for further processing.

\begin{tabular}{cc} 
Table 1. Dynamic characteristic parameter table based on the selection PCA \\
\hline Characteristic Parameters & Load \\
\hline Yaw_position & 131 \\
Int_tmp & 99 \\
Environment_tmp & 94 \\
Generator_speed & 78 \\
Power & 51 \\
\hline
\end{tabular}

\subsection{Feature Parameter Prediction by Elman Neural Network}

Given the slight fluctuations in the data set, it is of little significance for medium-term and long-term trend predictions. Therefore, all of the three types of data are continuously monitored for every ten-time series value, and the average value is one. That is, 39,480 pieces of data are reduced to 3,948 pieces of data, and subsequent experimental results prove that this can reduce the time complexity while grasping the large trend.

The effects predicted in real time by the Elman sub-predictive model trained based on historical monitoring data are shown in the following three sets of graphs. In the following three sets of figures, the top half of each set shows the predicted results from all the data in the excerpt time period. The left part of the lower part of the graph shows an enlarged view of the prediction results, and the right part shows the error between the predicted part and the actual monitored value. It can be seen from Figures 3(a) to 5(a) that the actual monitored values are close to the predicted values, and the deviation between real values and predictive values is in a small range, as shown in Figures 3(b) to 5(b). This indicates that the method used in this paper is feasible. The method proposed in this paper can start from 35,000, and after a few minutes of calculation, it can predict the following three types of monitoring data up to a value and trend of 39,870 and about 9.47 hours in advance. It is predicted that the prevention of icing in wind farms has good reference value.

\subsection{Multi-Source Data Fusion and BP Prediction}

In order to train based on the multi-source prediction data, it is possible to automatically determine whether there will be a problem of fan blade icing in the future. Each set of ten sets of data of these three types of data in the unfrozen running state and the data marked as the icing range are trained to train the BP neural network to achieve the purpose of self-cluster judgment. The BP self-clustering algorithm is trained by the 20 sets of data, and the icing or non-icing prediction based on data-driven multivariate data fusion is finally realized. 


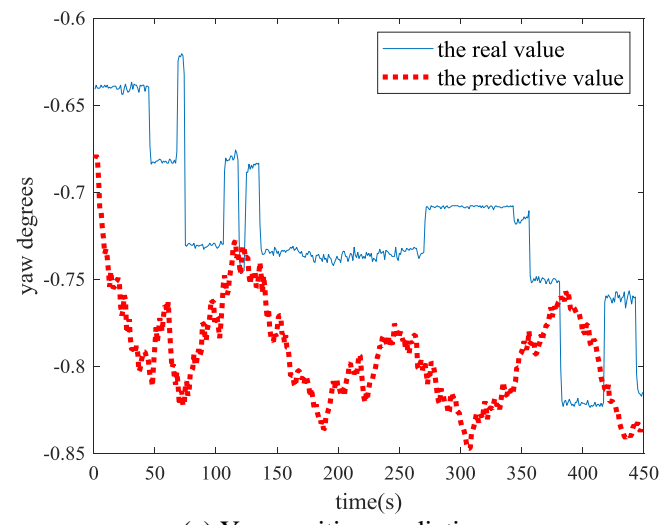

(a) Yaw position prediction curve

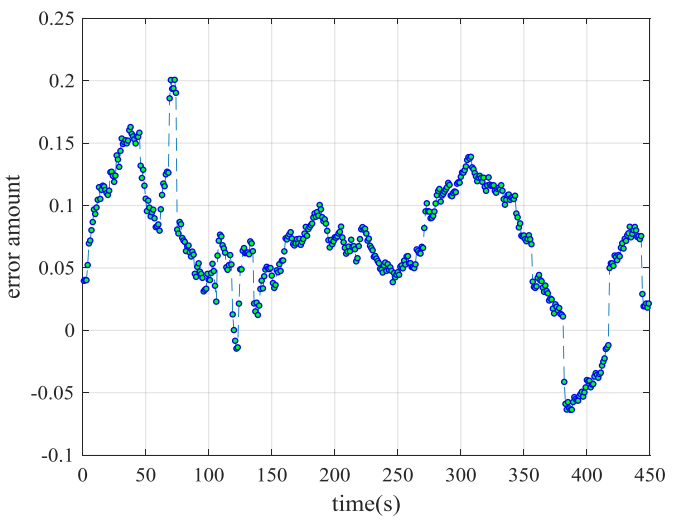

(b) Error amount

Figure 3. Yaw angle prediction results

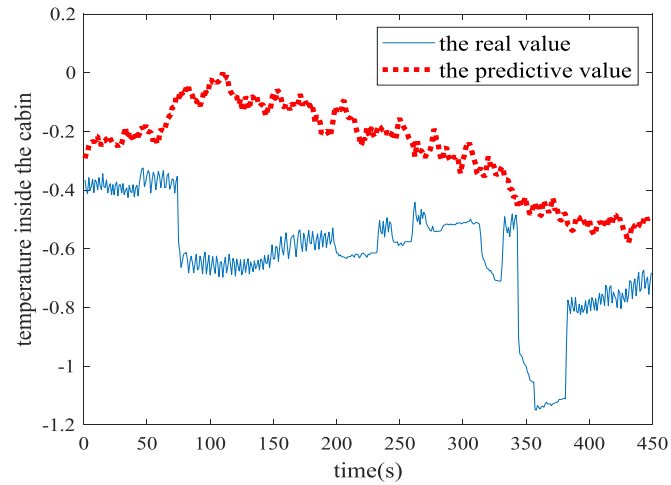

(a) Cabin temperature prediction curve

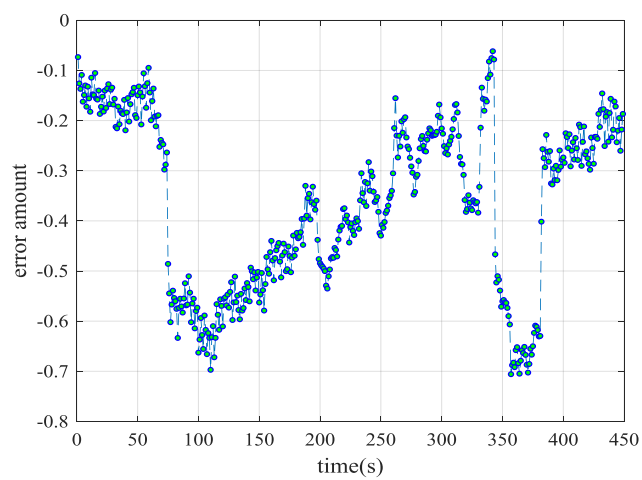

(b) Error amount

Figure 4. Cabin temperature prediction results

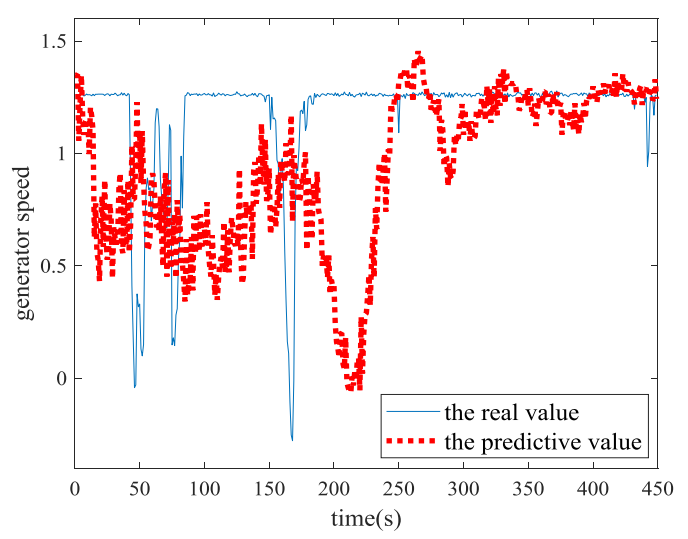

(a) Generator speed prediction curve

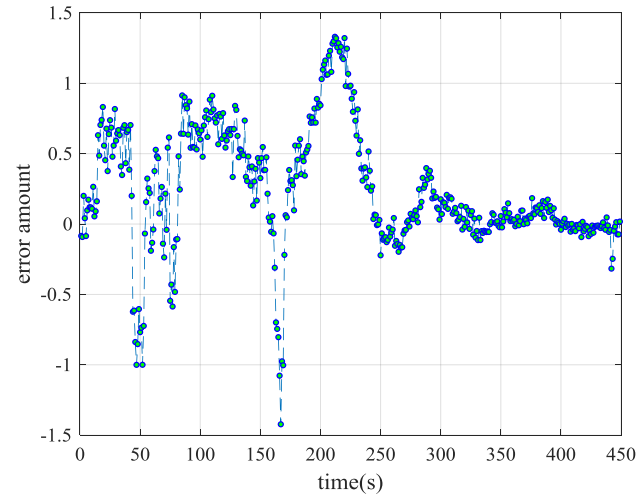

(b) Error amount

Figure 5. Generator speed prediction results

The number of hidden layer neurons determines the complexity and performance of the network and is also a key content of BP network design [19]. Refer to the empirical formula $n=\sqrt{n_{i}+n_{0}}+a$, where $n_{i}$ is the number of neurons in the input layer, $n_{0}$ is the number of neurons in the output layer, $n$ is the number of neurons in the hidden layer, and the range is from $[1,10]$. The number of nodes in the hidden layer calculated by the above formula is $[1,13]$. The mean square errors (MSE) of nine different hidden layer neural nodes are compared and analyzed. The network is used to perform multiple pieces of training to obtain the experimental results shown in the following figure. It can be seen that the minimum MSE and the minimum average MSE are BP networks with a neuron number of ten, and the minimum MSE training reaches 0.023 . This shows that the optimal number of hidden layer neurons should be eight.

According to the above analysis, the number of evaluation factors of the design network is five, the number of neurons in the input layer is six, the number of hidden layer neurons is eight, and the output layer node is one. The activation function of the hidden layer and the transfer function of the output layer use a logarithmic function (sigmoid) of 200 
iterations. Figure 6 is the mean square error of different hidden layer neurons.

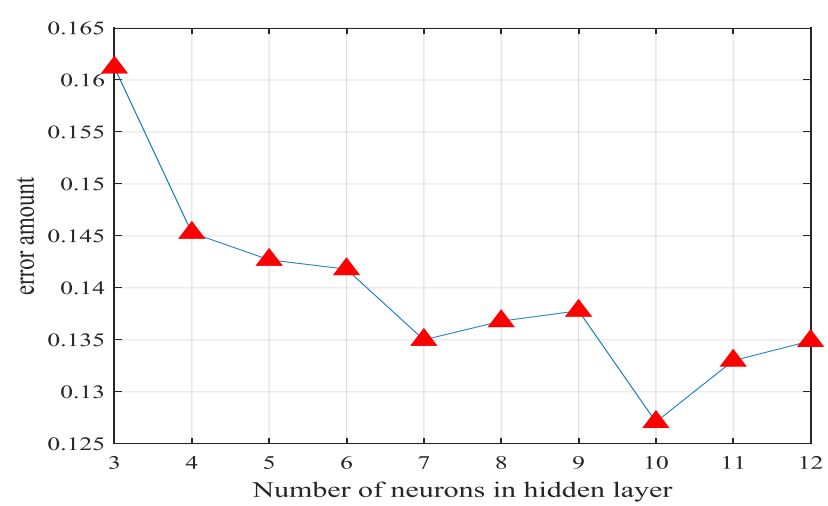

Figure 6. Mean square error of different hidden layer neurons

\section{Results Analysis}

\subsection{Diagnostic Performance Indicators}

In order to evaluate the accuracy of the proposed fault diagnosis model, the four statistical parameters are defined [20] as follows:

- TP (true positive): the number of times the wind turbine in a fault state is correctly diagnosed.

- FP (false positive): the number of times the wind turbine that is actually in a normal state is erroneously diagnosed as a fault state.

- FN (false negative): the number of times the wind turbine that is actually in a fault state is erroneously diagnosed as a normal state.

- TN (true negative): the number of times the wind turbine in a normal state is correctly diagnosed.

Then, we have Equations (5) to (8).

$$
\begin{gathered}
\text { Accuracy }=\frac{T P+T N}{T P+T N+T F+F N} \\
\text { Precision }=\frac{T P}{T P+F P} \\
\text { Recall }=\frac{T P}{T P+F N} \\
F 1=\frac{2 \times \text { Precision } \times \text { Recall }}{\text { Precision }+ \text { Recall }}
\end{gathered}
$$

\subsection{Analysis of Prediction Results}

In order to more accurately test the classification performance of the classifier and ensure that the classifier does not over-fit the data, the SCADA data is divided into training data $(70 \%)$ and verification data $(30 \%)$. The training data is used to train and optimize the model (in combination with ten-fold cross-validation), and the validation data is used to test the generalization performance of the model.

The basic performance indicators of each classifier are shown in the following Table 2. The results show that the classification performance of BP and SOM is significantly higher than that of SVM in the face of unbalanced data sets. In addition, although the accuracy of SOM can reach 0.938 , its precision and recall rate are both 0 , indicating that the classifier does not recognize the fault at all.

The performance indicators of each classifier combined with the SMOTE algorithm are shown in the following Table 3. 
The results show that the classification performance of each classifier is improved after the data set is balanced using the SMOTE algorithm.

\begin{tabular}{ccccc}
\multicolumn{5}{c}{ Table 2. Classifier basic performance indicators } \\
\hline Algorithm & Accuracy & Precision & Recall & F1 \\
BP & 0.984 & 0.929 & 0.850 & 0.886 \\
SOM & 0.938 & 0.000 & 0.0000 & 0.000 \\
SVM & 0.959 & 0.874 & 0.246 & 0.380 \\
\hline & & & & \\
& Table 3. Combined with the performance indicators of the SMOTE+Tomek links algorithm & F1 \\
\hline Algorithm & Accuracy & Precision & $99.70 \%$ & $98.88 \%$ \\
SOM & $98.89 \%$ & $97.97 \%$ & $7.79 \%$ & $72.81 \%$ \\
SVM & $69.69 \%$ & $65.80 \%$ & $74.63 \%$ & $76.25 \%$ \\
\hline
\end{tabular}

According to the above experiments, BP has the best diagnostic performance, so this paper uses this algorithm to construct a blade icing failure prediction model.

\section{Conclusions}

The paper innovatively proposes a data-driven wind turbine fault icing prediction method. The specific conclusions are as follows: the icing failure prediction algorithm proposed by the three-source data fusion in this paper can predict the icing failure of the wind turbine blade earlier, and it can be more fault-tolerant than single-source data prediction. It is also better at excavating the industrial standardization that is formed and exploring the value of SCADA data sets that form industrial standardization. Due to the current constraints on data sharing in the wind energy industry, the data available for research is very limited. The models proposed in practical engineering applications need to be tested on a wider data set for further optimization.

\section{Acknowledgements}

This paper is supported by the Natural Science Foundation of China (No. 61771492, 61871432), the Natural Science Foundation of Hunan Province (No. 2017JJ3065, 2019JJ60054, and 2019JJ60008), the 2018 China Scholarship Council Higher Education Teaching Method Research Project, and the 2017 Zhuzhou Science and Technology Project.

\section{References}

1. M. Anuradha, B. K. Keshavan, T. S. Ramu, and V. Sankar, "Probabilistic Modeling and Forecasting of Wing Power," International Journal of Performability Engineering, Vol. 12, No. 4, pp. 353-368, 2016

2. Z. Q. Zhai, C. Lin, H. M. Cui, H. Y. Liang, and H. Y. Cheng, "Fatigue Life Prediction for Throwing Impeller of An Impeller Blower," International Journal of Performability Engineering, Vol. 15, No. 1, pp. 127-137, 2019

3. H. Y. Fan, P. K. Tarun, V. C. P. Chen, D. T. Shih, J. M. Rosenberger, S. B. Kim, et al., "Data-Driven Optimization for Dallas Fort Worth International Airport Deicing Activities," Annals of Operations Research, Vol. 263, No. 1-2, pp. 361-384, April 2018

4. Y. Zhao, J. M. Feng, Q. Zhou, and X. Y. Peng, "Blade Fracture Analysis of a Motor Cooling Fan in a High-Speed Reciprocating Compressor Package," Engineering Failure Analysis, Vol. 89, pp. 88-99, July 2018

5. A. Alzghoul, M. Lfstrand, and B. Backe, "Data Stream Forecasting for System Fault Prediction," Computers and Industrial Engineering, Vol. 62, No. 4, pp. 972-978, May 2012

6. A. Kusiak and A. Verma, "A Data-Mining Approach to Monitoring Wind Turbines," IEEE Transactions on Sustainable Energy, Vol. 3, No. 1, pp. 150-157, 2012

7. T. Zhang, "Design and Implementation of Wind Turbine Blade Condition Monitoring and Fault Diagnosis System," Shanghai Institute of Electrical Engineering, 2016

8. Q. Shi, "Model-based Detection for Ice on Wind Turbine Blades," NTNU, 2017

9. Y. H. Cao, J. M. Guo, Y. Li, and H. Q. Lv, "Engine Life Prediction based on Degradation Data," International Journal of Performability Engineering, Vol. 14, No. 12, pp. 2905-2914, 2018

10. R. Chandra, "Competition and Collaboration in Cooperative Convolution of Elman Recurrent Neural Networks for Time-Series Prediction," IEEE Transactions on Neural Networks and Learning Systems, Vol. 26, No. 12, pp. 3123-3136, December 2015

11. J. Bilski and J. Smoląg, "Parallel Architectures for Learning the RTRN and Elman Dynamic Neural Networks," IEEE Transactions on Parallel and Distributed Systems, Vol. 26, No. 9, pp. 2561-2570, September 2015

12. T. Y. Wu, C. Y. Zhang, and Y. S. Zhao, "Monitoring and Warning Methods of Tailings Reservoir using BP Neural Network," International Journal of Performability Engineering, Vol. 14, No. 6, pp. 1171-1180, June 2018

13. Z. W. Shang, X. Liu, X. X. Liao, R. Geng, M. S. Gao, and J. T. Yun, "Rolling Bearing Fault Diagnosis Method based on EEMD and GBDBN," International Journal of Performability Engineering, Vol. 15, No. 1, pp. 230-240, 2019 
14. S. Jia, Q. Qiu, J. Li, Y. Li, and Y. Cong, "BP Neural Network based Localization for a Front-Wheel Drive and Differential Steering Mobile Robot," in Proceedings of IEEE International Conference on Information and Automation, pp. 2270-2274, Lijiang, October 2015

15. Y. Ge, L. Z. Guo, and Y. Dou, "Remaining Useful Life Prediction of Machinery based on K-S Distance and LSTM Neural Network," International Journal of Performability Engineering, Vol. 15, No. 3, pp. 895-901, 2019

16. B. H. Zheng, "Material Procedure Quality Forecast based on Genetic BP Neural Network," Modern Physics Letters, Vol. 31 , No. $19-21,2017$

17. H. H. Kim and N. R. Swanson, "Mining Big Data using Parsimonious Factor, Machine Learning, Variable Selection and Shrinkage Methods," International Journal of Forecasting, Vol. 34, No. 2, pp. 339-354, 2018

18. J. X. Bi, C. L. Zheng, H. Z. Huang, Y. Zhou, and X. X. Li, "Load Analysis and Calculation Optimization of Horizontal Axis Wind Turbine Blades," International Journal of Performability Engineering, Vol. 14, No. 12, pp. 3098-3108, December 2018

19. Y. X. Zhang, X. D. Gao, and S. Katayama, "Weld Appearance Prediction with BP Neural Network Improved by Genetic Algorithm During Disk Laser Welding,” Journal of Manufacturing Systems, Vol. 34, pp. 53-59, 2015

20. L. Luo, R. J. Lovelett, and B. A. Ogunnaike, "Hierarchical Monitoring of Industrial Processes for Fault Detection, Fault Grade Evaluation, and Fault Diagnosis,” AIChE Journal, Vol. 63, pp. 2781-2795, 2017

Cheng Peng received his M.S. and PhD. degrees from the School of Information Science and Engineering at Central South University in 2010 and 2013, respectively. He is currently an associate professor. His current research interests include industry big data analysis and industry equipment health management.

Jing He is a master's student in the School of Computer Science at Hunan University of Technology. Her main research interest is industry equipment health management.

Hao Chi is a master's student in the School of Computer Science at Hunan University of Technology. His main research interest is industry equipment health management.

Xinpan Yuan is an instructor in the School of Computer Science at Hunan University of Technology. His current research interests include industry big data analysis and industry equipment health management.

Xiaojun Deng is a professor in the School of Computer Science at Hunan University of Technology. His current research interests include industry big data analysis and industry equipment health management. 